\title{
Hyperspectral Reflectance Characteristics of Cyanobacteria
}

\author{
Terrence Slonecker*, Brittany Bufford, Jennifer Graham, Kurt Carpenter, Dan Opstal, \\ Nancy Simon, Natalie Hall
}

US Geological Survey, Reston, VA, USA

Email: ^tslonecker@usgs.gov

How to cite this paper: Slonecker, T., Bufford, B., Graham, J., Carpenter, K., Opstal, D., Simon, N. and Hall, N. (2021) Hyperspectral Reflectance Characteristics of Cyanobacteria. Advances in Remote Sensing, 10, 66-77.

https://doi.org/10.4236/ars.2021.103004

Received: July 14, 2021

Accepted: August 23, 2021

Published: August 26, 2021

Copyright $\odot 2021$ by author(s) and Scientific Research Publishing Inc. This work is licensed under the Creative Commons Attribution International License (CC BY 4.0).

http://creativecommons.org/licenses/by/4.0/

\begin{abstract}
Potentially harmful cyanobacterial blooms are an emerging environmental concern in freshwater bodies worldwide. Cyanobacterial blooms are generally caused by high nutrient inputs and warm, still waters and have been appearing with increasing frequency in water bodies used for drinking water supply and recreation, a problem which will likely worsen with a warming climate. Cyanobacterial blooms are composed of genera with known biological pigments and can be distinguished and analyzed via hyperspectral image collection technology such as remote sensing by satellites, airplanes, and drones. Here, we utilize hyperspectral microscopy and imaging spectroscopy to characterize and differentiate several important bloom-forming cyanobacteria genera obtained in the field during active research programs conducted by US Geological Survey and from commercial sources. Many of the cyanobacteria genera showed differences in their spectra that may be used to identify and predict their occurrence, including peaks and valleys in spectral reflectance. Because certain cyanobacteria, such as Cylindrospermum or Dolichospermum, are more prone to produce cyanotoxins than others, the ability to differentiate these species may help target high priority waterbodies for sampling. These spectra may also be used to prioritize restoration and research efforts to control cyanobacterial harmful algal blooms (CyanoHABs) and improve water quality for aquatic life and humans alike.
\end{abstract}

\section{Keywords}

Cyanobacterial Harmful Algal Blooms (CyanoHABs), Cyanobacteria, Hyperspectral Remote Sensing, Hyperspectral Microscopy, Imaging Spectroscopy

\section{Introduction}

Freshwater cyanobacterial harmful algal blooms, or cyanoHABs are a worldwide 
concern. The growth and extent of potentially toxic cyanobacterial blooms are not immediately distinguishable from non-toxic blooms. Potentially harmful cyanoHABs bacterial blooms have recently been exacerbated by high nutrient inputs and warmer waters.

CyanoHABs have been appearing with increasing frequency in water bodies used for drinking water supply and recreation, a problem which will likely worsen as the climate warms [1] [2]. CyanoHAB bacterial blooms may cause unsightly surface scums, produce taste-and-odor causing compounds, and some strains of cyanobacteria produce potent cyanotoxins which are hazardous to human and animal health [3]. Species identification will give an indication of whether or not potentially toxic organisms are present, but the only way to definitively determine whether or not a bloom is toxic is to test for toxins [4]. This work shows that overhead imagery from satellites and aircraft have the potential to monitor and calibrate the level of danger to human and animal life. Further research is needed.

Remote sensing has traditionally been used to monitor the intensity and extent of CyanoHAB phenomena as the biological pigments involved lend themselves to detection by multispectral remote sensing systems such as the US Geological Survey's (USGS) Landsat or the European Space Agency's (ESA) Sentinel 2/3 imaging satellites. However, the development of hyperspectral remote sensing systems promises to move the science of monitoring CyanoHABs beyond algal or cyanobacterial presence, estimates of abundance, [5] and geographic extent into the realm of chemical analysis at the cellular level. With the advent of new satellite systems, such as the DESIS (DLR Earth Sensing Imaging Spectrometer), now on the International Space Station [6] and HICO (Hyperspectral Imager for the Coastal Ocean) [7] as well as the expansion of the Advanced Visible Infrared Imaging Spectrometer (AVIRIS) program at the National Aeronautics and Space Administration (NASA), and the emergence of small hyperspectral sensors for Unmanned Aerial Vehicles (UAVs), hyperspectral technology promises to advance earth systems monitoring to a new level [8].

All phytoplankton are composed of mixtures of photosynthetic pigments that can, in many cases, be distinguished by spectroscopic techniques. Here, utilizing a hyperspectral microscope, we document the spectral characteristics of 13 common genera of cyanobacteria to demonstrate the potential identification capabilities of hyperspectral remote sensing technology.

CyanoHABs are one of the emerging climate change issues that are currently drawing interest from both the civilian and intelligence remote sensing communities. Obviously related to ecosystem health and suitability of water quality for a range of uses, CyanoHABs also carry relevant attention to the defense and intelligence communities [9] [10] in that large coastal populations may suddenly be denied a major food source as a result of CyanoHAB contamination, potentially destabilizing large populations.

For many years, remote sensing has been used to map algal blooms. Mapping of chlorophyll-a is widely used as an indicator of water quality, but because both 
eukaryotic algae and cyanobacteria contain chlorophyll-a, it can suffer from a lack of specificity and is most successful in blooms that are known to be dominated by cyanobacteria [11]. An alternative is using the accessory pigment phycocyanin, produced by cyanobacteria but not by many other phytoplankton, as a proxy [10] [11] However, methods that measure phycocyanin in the lab are not standardized, and phycocyanin is less easily detected with remote sensing [11]. Another approach is the Cyanobacteria Index (CI), which uses the absorption feature at $681 \mathrm{~nm}$ to distinguish cyanobacteria from eukaryotic algae [11]. Chlorophyll-a in eukaryotic algae fluoresces at $681 \mathrm{~nm}$, but chlorophyll-a in cyanobacteria is contained in Photosystem I and does not fluoresce, so the signal at this wavelength is dominated by chlorophyll absorption and the difference in spectral shape can be quantified to determine the type of algae present [11].

Recently, there has been an increase in the availability of hyperspectral imaging sensors, which can measure more than thirty narrow bands (usually more than 100 bands) to produce a complete, high-resolution spectrum. These spectra may reveal details that were previously invisible to sensors with fewer bands, opening a path to the development of new models that consider the entire spectrum. Kudela et al. [12] made use of a spectral shape algorithm to discriminate Aphanizomenon, a non-toxic genus in many waterbodies, from Microcystis in California lakes. The Aphanizomenon-Microcystis Index uses a ratio of the width of the major chlorophyll a reflectance feature to the width of the phycocyanin absorption feature and was developed using aerial hyperspectral imagery. The algorithm provides an estimate of the relative abundance of Microcystis and Aphanizomenon in a pixel and was calibrated with measured cell counts [10].

The high-resolution spectra provided by hyperspectral imaging sensors also suggests that other analysis techniques, such as the spectral derivative, can yield further details. Hunter [13] used first-derivative analysis to discriminate between phytoplankton color groups, although the study was not particularly focused on separating cyanobacterial genera [13]. The spectral derivative accentuates differences between spectra that might not otherwise be apparent, providing a useful tool for the separation of spectrally similar species.

\section{Materials and Methods}

Water and CyanoHAB samples were collected by various USGS Water Science Centers, and extra samples were also purchased from Carolina Biological Supply, Burlington, NC, a commercial biological materials company. Split samples were shipped to the USGS water labs in Reston, Virginia, and the National Institute of Standards and Technology (NIST) in Gaithersburg, Maryland. At NIST, samples were prepared on wet slides and imaged and analyzed on a hyperspectral microscope.

The hyperspectral microscope consists of two parts: the microscope component is an Olympus MVX-10 microscope with an MX-2X Objective and a $1-4 \times$ magnification changer, and the hyperspectral imaging component is a Surface 
Optics SOC-710 hyperspectral camera (Surface Optics Corporation, San Diego, Calif)*. The SOC-710 measures 128 spectral bands, at $4.69 \mathrm{~nm}$ spectral sampling over the $400 \mathrm{~nm}$ to $1000 \mathrm{~nm}$ spectral range. The finest spatial resolution of the system is on the order of $0.2 \mu \mathrm{m} /$ pixel. Illumination was provided by three 35-watt quartz-halogen lamps set with dichroic reflectors, with a high color temperature, in order to more closely match the top-of-atmosphere (TOA) solar spectrum. The geometry approximates a $45^{\circ}$-incident and $0^{\circ}$-observation arrangement. This is not a strictly defined geometry due to the lamp projection angle, lens collection angle, and interreflections between the sample surface and the objective lens.

Surface algal samples were collected by hand or by using forceps or tweezers and stored in $70 \mathrm{~mm}$-vials for storage and transport. Samples from commercial labs were collected from the top of the vial or test-tube used for shipping. Both commercial and field samples were opened, and large clumps of algae were removed, spread over a glass slide to make as uniform a surface as possible, then covered with a glass cover.

Before algal sample measurement, reference data cubes were collected from the reference slide placed on top of a Spectralon ${ }^{\circledR}$ disk (Labsphere, Inc., North Sutton, N.H.), and integration times were optimized for each desired magnification level. Data cubes were then collected from the sample slides. Depending on integration time, image acquisition took between 2 and 15 minutes to complete. Two to ten images were collected per slide, depending on algal abundance. A fresh reference slide was measured after data collection was finished. Slides were kept out of direct light when not being measured. Data cubes were normalized using commercial image processing software (ENVI-Environment for Visualizing Images, Harris Geospatial Solutions), Boulder, Colorado, to the reference data cube with the corresponding magnification and integration time. Normalization to the entire reference cube (dividing each image pixel by the corresponding pixel of the reference cube) eliminated some detector artifacts and noise that occurred in the images, especially at higher magnifications [14]. Normalization was achieved by:

$$
R=(s 1-s 2) /(s 3-s 2)
$$

where $R$ is the reflectance, $s 1$ is the sample image cube signal, $s 2$ is the dark reference cube signal, and $s 3$ is the white reference signal. Due to interreflections between the coverslip and the sample, adjacency effects, and the large degree of variation in cell density, the resulting reflectance was normalized to one. the absolute reflectance of algae is low (on the order of $10 \%$ or less). The results reported are the normalized hyperspectral image cubes in relative reflectance. Pixels were selected manually, as regions of interest, to represent the algal cells and reduce the empty background space. The spectra reported are the averages of over 100 pixels. The measurement error is a subject for further research and will address the complexities of microscopic spectroscopy. We expect the relative 
shape of the spectra to remain consistent.

The first derivative spectra for each sample is also available in the ScienceBase data release for this project (available at: https://doi.org/10.5066/P9SY4PSK). Derivative spectra are valuable in spectrographic studies as they often highlight features less apparent in zero-order spectra. The first-order derivative represents the rate of change of reflectance with respect to wavelength. The first-order spectral plot also passes through zero at the same wavelength as the peak of the reflectance (or absorbance) band. More importantly, similar plant species that have nearly identical zero-order reflectance spectra, often have unique and/or distinctive first-order spectra. Table 1 below shows the CyanoHAB genera identified, along with their spectral reflectance features.

Also available are flat-field corrections for each spectral graphic. Designated with a "FF" in the title individual files, Flat-field correction is a calibration process that produces relative reflectance by dividing each pixel by a "flat" spectrum of low-reflectance part of the image, usually designated by a small part of the image known as a region of interest, or ROI. The flat spectrum is usually something uniform and spectrally low in reflectance, like concrete or sand. One of the big advantages of the flat-field correction is that produces a uniform image with y-axis units in percent reflectance, which is generally required for spectral libraries.

Algal genera examined in this project and their spectral reflectance features. Most samples were obtained from a commercial biological supply company (Carolina Biological Supply, Burlington, North Carolina).

Table 1. Features of selected genera of cyanobacteria spectra.

\begin{tabular}{|c|c|c|}
\hline Genera & Source & Reflectance features (nm) (Pk: peak, Ab: absorption) \\
\hline Aphanizomenon & Field & Pk: 554.9, Ab: 674.8, Pk: 701.1 \\
\hline Cylindrospermum & Lab & Pk: 534.3, Ab: 627.6, Pk: 643.2, Ab: 674.8, Pk: 706.4 \\
\hline Dolichosperm & Both & Pk: 529.2, Ab: 627.6, Pk: 643.2, Ab: 674.8, Pk: 706.4, Ab: 743.6 \\
\hline Eucapsis & Lab & $\begin{array}{l}\text { Pk: 524.1, Ab: 534.3, Pk: 544.6, Ab: 617.1, Pk: 643.2, Ab: 669.5, } \\
\text { Pk: 701.1 }\end{array}$ \\
\hline Gloeocapsa & Lab & Pk: 529.2 \\
\hline Gloeotrichia & $\mathrm{Lab}$ & $\begin{array}{l}\text { Pk: 513.8, Pk: 529.2, Ab: 565.2, Pk: 585.9, Ab: 622.3, Pk: 643.2, } \\
\text { Ab: 674.6, Pk: 706.4 }\end{array}$ \\
\hline Lyngbya & $\mathrm{Lab}$ & Pk: 534.3, Ab: 627.6, Pk: 648.5, Ab: 674.8, Pk: 711.7 \\
\hline Merismopedia & $\mathrm{Lab}$ & Pk: 529.2, Ab: 627.6, Pk: 643.2, Ab: 674.8, Pk: 690.6 \\
\hline Microcystis & Both & Pk: 549.8, Ab: 674.8,Pk: 685.3 \\
\hline Nostoc & Lab & Pk: 529.2, Ab: 539.5; Pk: 549.8 \\
\hline Oscillatoria & Lab & Pk: 534.3, Ab: 627.6, Pk: 648.5, Ab: 669.5, Pk: 711.7 \\
\hline Spirulina & Lab & $\begin{array}{l}\text { Pk: 529.2, Ab: 539.5, Pk: 549.8, Ab: 627.6, Pk: 643.2, Ab: 674.8, } \\
\text { Pk: 701.1, Ab: 738.3, Pk: 759.6 }\end{array}$ \\
\hline Tolypothrix & Lab & Pk: 529.2, Ab: 539.5, Ab: 627.6, Pk: 643.2, Ab: 674.8, Pk; 706.4 \\
\hline
\end{tabular}




\section{Results}

Cyanobacteria such as those commonly known to be CyanoHABs, are a phylum of single-celled bacteria that produce oxygen through photosynthesis. They are generally characterized by blue-green pigments (phycobiliproteins) and often form colonies that are linear filaments, sheets, or spheres (Figure 1).

The following (Figures 2-6) represents a collection of hyperspectral data cubes, displayed as RGB images and spectral plots, of some of the most common forms of cyanobacteria/cyanoHABs. Hyperspectral imaging in the laboratory permits controlled characterization of spectral reflectance at the cellular level without any complication from atmospheric or other water constituents, such as colored dissolved organic matter (CDOM and or/detritus/sediment) interferences. Only selected examples are shown here. The full dataset of $13 \mathrm{HAB}$ genera is available for download in ScienceBase at: https://doi.org/10.5066/P9SY4PSK.

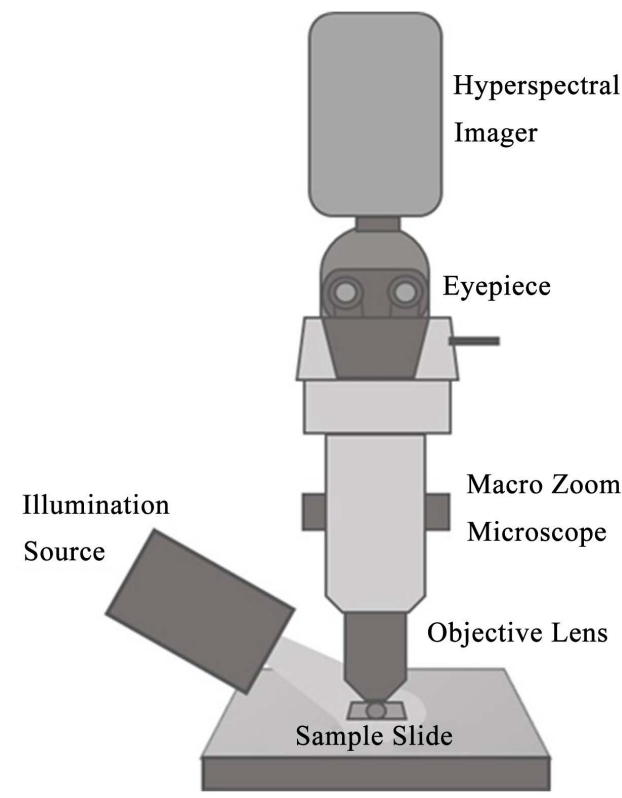

Figure 1. The hyperspectral microscope consists of two parts: 1) the microscope component is a macro-zoom microscope with a $2 \times$ objective lens, and a $1-4 \times$ camera-magnification changer coupled with, 2) a hyperspectral imager. Source: D. Allen.
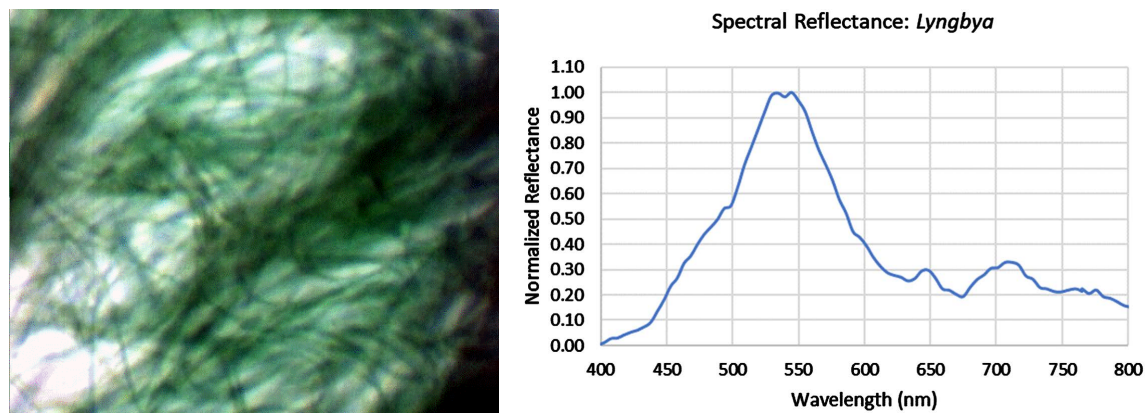

Figure 2. Hyperspectral image and spectra of the genus Lyngbya. Lyngbya is characterized by a large, reflectance with a peak around $550 \mathrm{~nm}$, another around $648.5 \mathrm{~nm}$, and a third peak at $711.7 \mathrm{~nm}$. 



Figure 3. Hyperspectral image and spectra of Gloeocapsa which is characterized by a similar large, broad reflectance with a peak around $535 \mathrm{~nm}$.
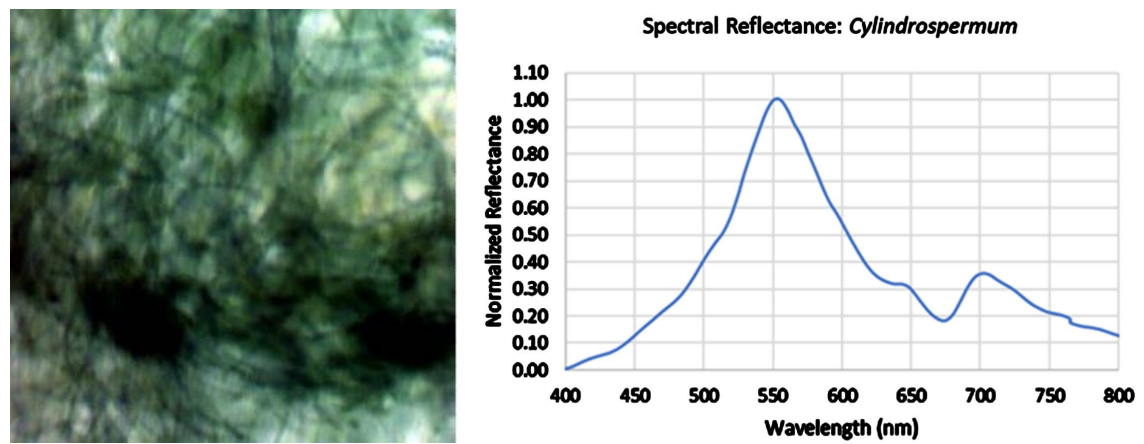

Figure 4. Hyperspectral image and spectra of Gloeocapsa which is characterized by a similar large, broad reflectance with a peak around $535 \mathrm{~nm}$.


Figure 5. Hyperspectral Image and spectra of Gloeotrichia, showing three large peaks between $500 \mathrm{~nm}$ to $700 \mathrm{~nm}$.
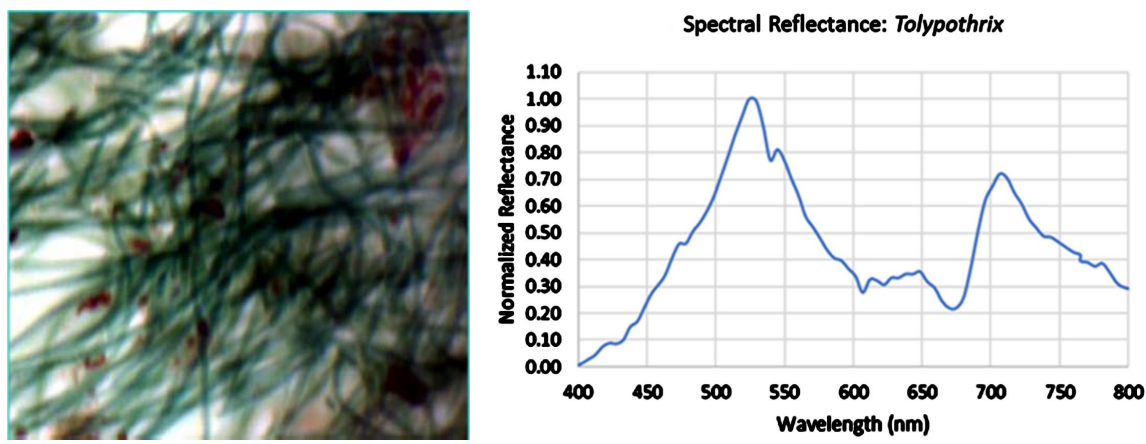

Figure 6. Hyperspectral image and spectra of Tolypothrix, showing two dominant peaks around 500 and $700 \mathrm{~nm}$. 
Spectral Reflectance: Cylindrospermum

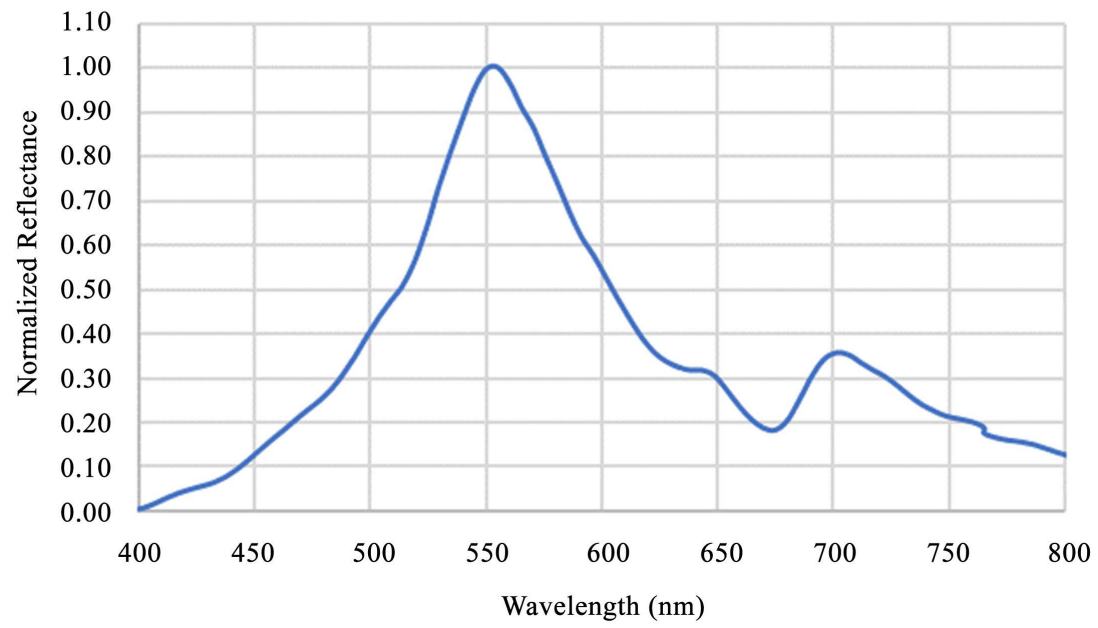

(a)

Spectral Reflectance: Gloeocapsa

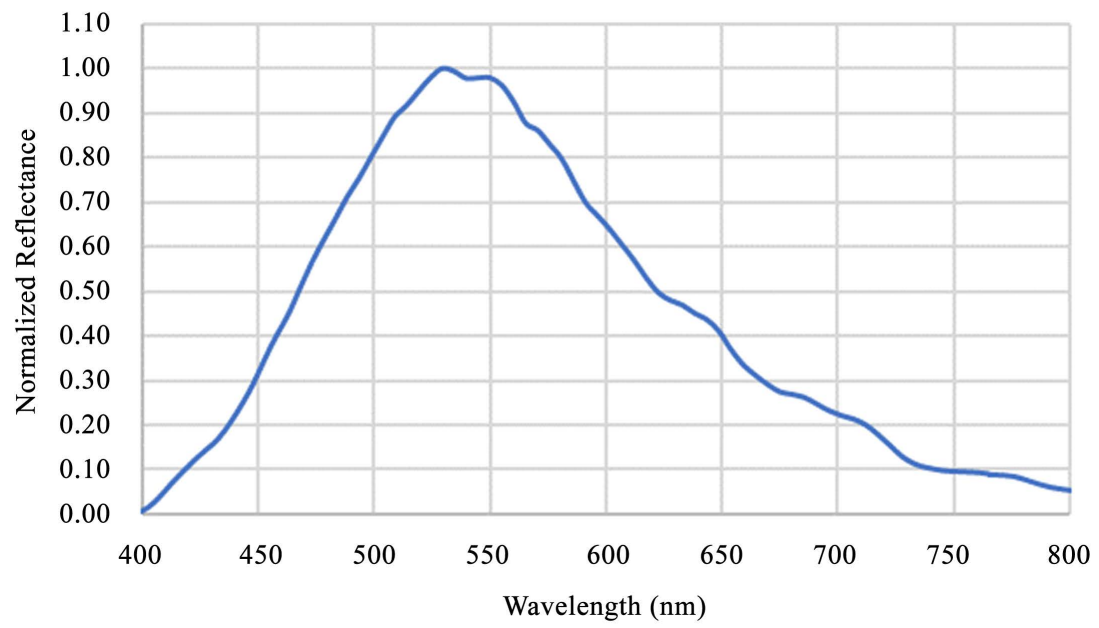

(b)

First Derivative of Reflectance: Cylindrospermum



Wavelength (nm)

(c) 
First Derivative of Reflectance: Gloeocapsa



(d)

Figure 7. The similar spectral reflectance of Cylindrospermum and Gloeocapsa ((a) and (b)). The same spectra for each species transformed into the first derivative ((c) and (d)). The basic spectral plots are very similar while the derivative plots are unique with resp Figure 7. The similar spectral reflectance of Cylindrospermum and Gloeocapsa ((a) and (b)). The same spectra for each species transformed into the first derivative ((c) and (d)). The basic spectral plots are very similar while the derivative plots are unique with respect to dominant features. Note: the finer features are likely representations of noise.

As might be expected, some of the hyperspectral signatures from CyanoHAB genera are very similar with only minor variations. A common HAB is Lyngbya which is shown in Figure 2 along with its visible, near, infrared (VNIR) hyperspectral plot. The spectra were taken from the hyperspectral image on a dominant patch of algae near the center of the image.

Figure 5 and Figure 6 show the VNIR reflectance profile of two unique algae genera, Gloeotrichia and Tolypothrix that are generally associated with harmful blooms and occasional toxin production. Some species have the same basic broad peak but have a second smaller peak around $700 \mathrm{~nm}$. Some CyanoHABs present very unusual variations on the basic CyanoHAB spectra such as Gloeotrichia and Tolypothrix where dominant peaks are located at different points in the spectrum. See Table 1.

The common data transformation utilized here is the spectral derivative (see Figure 7). The first-order derivative is a standard spectroscopic transformation that plots the rate of change of absorbance or reflectance with respect to the rate of change with respect to wavelength. Commonly known as "rise over run", it is noted as $\mathrm{dR} / \mathrm{d} \lambda$. Calculation of the first derivative is a common, often-practiced method of separating noise from signal in spectroscopic applications. In applications relating to vegetation spectra, reflectance curves of different plant species are often very similar and difficult to differentiate in standard reflectance libraries but when the first derivative transformations are employed, individual species have much more unique and separable spectral signatures. As shown above, this is also the case for the samples analyzed and can potentially be used for spe- 
cies differentiation.

\section{Summary}

In this short communication, we seek to make three basic points. First, cyanobacteria that comprise most CyanoHABs are composed of pigments that have spectrally strong signatures in the visible and near infrared (VNIR) portion of the spectrum and generally unique spectral signatures, in the basic reflectance, or first derivative transformations, at the genus taxonomic level. A second point is that cyanobacteria generally have distinct signatures that could allow identification of certain types, such as Lyngbya or Dolichospermum, that are likely to produce toxins. Third, the growing availability of hyperspectral imagery could create new analytical techniques that involve stand-off, overhead remote sensing, augmenting in-situ and laboratory chemical analysis.

Although this work is preliminary, it demonstrates a change in remote sensing where information extracted from imagery is based not only morphology, but also on a spectral profile. The hyperspectral imagery utilized here, although from a microscope, is the same electromagnetic energy that is captured by orbital platforms such as DESIS and PRISMA. Measuring biology from space may soon be part of a changing remote sensing paradigm.

\section{Acknowledgements}

Acknowledgement to David Allen (NIST) for his involvement in the conceptualization and methodology development for this project, as well as the spectral laboratory processing.

\section{Author Contributions}

T. Slonecker and J. Graham were involved in the project conceptualization, methodology development and writing. N. Simon and K. Carpenter were involved in the project conceptualization and data collection. B. Bufford was responsible for sample processing. Natalie Hall was involved with overall project management and writing.

Special thanks to Dr David Allen of NIST who was instrumental to this project.

\section{Funding}

This research was funded by the Water Mission Area and the National Civil Applications Center of the National Land Imaging Program of the US Geological Survey. Work at the National Institute of Standards and Technology was accomplished under Interagency Agreement between the National Institute of Standards and Technology (NIST) and the US Geological Survey (USGS) number G19PG00046.

\section{Disclaimer}

Any use of trade, firm, or product names is for descriptive purposes only and 
does not imply endorsement by the US Government. All images and figures in this work were obtained through Interagency Agreement between the National Institute of Standards and Technology (NIST) and the US Geological Survey (USGS) number G19PG00046.

\section{Conflicts of Interest}

The authors declare no conflict of interest. The funders had no role in the design of the study; in the collection, analyses, or interpretation of data; in the writing of the manuscript, or in the decision to publish the results.

\section{References}

[1] O’Neil, J., Davis, T.W., Burford, M.A. and Gobler, C.J. (2012) The Rise of Harmful Cyanobacteria Blooms: The Potential Roles of Eutrophication and Climate Change. Harmful Algae, 14, 313-334. https://doi.org/10.1016/j.hal.2011.10.027

[2] Paerl, H.W., et al. (2001) Harmful Freshwater Algal Blooms, with an Emphasis on Cyanobacteria. Scientific World Journal, 1, 76-113.

[3] Carmichael, W.W. (2001) Health Effects of Toxin-Producing Cyanobacteria: "The CyanoHABs”. Human and Ecological Risk Assessment: An International Journal, 7, 1393-1407.

[4] Graham, J., et al. (2008) Cyanobacteria in Lakes and Reservoirs: Toxin and Tasteand-Odor Sampling Guidelines (Ver. 1.0): US Geological Survey Techniques of Water-Resources Investigations, Book 9, Chap. A7, Section 7.5. Reworded the Conclusion.

[5] Leegleiter, C.J., Stegman, T.K. and Overstreet, B.T. (2016) Spectrally Based Mapping of Riverbed Composition. Geomorphology, 264, 61-79. https://doi.org/10.1016/j.geomorph.2016.04.006

[6] Eckardt, A., et al. (2015) DESIS (DLR Earth Sensing Imaging Spectrometer for the ISS-Muses Platform). 2015 IEEE International Geoscience and Remote Sensing Symposium (IGARSS), Milan, 26-31 July 2015, 1457-1459.

[7] Lucke, R.L., et al. (2011) Hyperspectral Imager for the Coastal Ocean: Instrument Description and First Images. Applied Optics, 50, 1501-1516.

https://doi.org/10.1364/AO.50.001501

[8] Fichot, C.G., et al. (2016) High-Resolution Remote Sensing of Water Quality in the San Francisco Bay-Delta Estuary. Environmental Science \& Technology, 50, 573-583. https://doi.org/10.1021/acs.est.5b03518

[9] Jewett, E., et al. (2008) Harmful Algal Bloom Management and Response: Assessment and Plan.

[10] Paine, E.C., et al. (2018) Optical Characterization of Two Cyanobacteria Genera, Aphanizomenon and Microcystis, with Hyperspectral Microscopy. Journal of Applied Remote Sensing, 12, Article ID: 036013.

[11] Stumpf, R.P. and Tomlinson, M.C. (2007) Remote Sensing of Harmful Algal Blooms. In: Remote Sensing of Coastal Aquatic Environments, Springer, Berlin, 277-296.

[12] Kudela, R.M., et al. (2015) Application of Hyperspectral Remote Sensing to Cyanobacterial Blooms in Inland Waters. Remote Sensing of Environment, 167, 196-205. https://doi.org/10.1016/j.rse.2015.01.025

[13] Hunter, P.D., et al. (2008) Spectral Discrimination of Phytoplankton Colour Groups: 
The Effect of Suspended Particulate Matter and Sensor Spectral Resolution. Remote Sensing of Environment, 112, 1527-1544. https://doi.org/10.1016/j.rse.2007.08.003

[14] Xue, L. (2010) Application of IDL and ENVI Redevelopment in Hyperspectral Image Preprocessing. In: International Conference on Computer and Computing Technologies in Agriculture, Springer, Berlin, 403-409.

\section{Supplementary Materials}

All data for this project are available in Sciencebase at https://doi.org/10.5066/P9SY4PSK. 